\title{
Peptide Receptor Radionuclide Therapy for the Treatment of Pancreatic Neuroendocrine Tumors: Recent Insights
}

This article was published in the following Dove Press journal: OncoTargets and Therapy

\author{
Jason S Starr (D) \\ Mohamad Bassam Sonbol (iD) ${ }^{2}$ \\ Timothy J Hobday ${ }^{3}$ \\ Akash Sharma (D) ${ }^{4}$ \\ Ayse Tuba Kendi ${ }^{3}$ \\ Thorvardur R Halfdanarson (1D $)^{3}$ \\ 'Division of Hematology/Oncology, Mayo \\ Clinic, Jacksonville, FL, USA; ${ }^{2}$ Division of \\ Hematology/Oncology, Mayo Clinic, \\ Phoenix, AZ, USA; ${ }^{3}$ Division of \\ Hematology/Oncology, Mayo Clinic, \\ Rochester, MN, USA; ${ }^{4}$ Division of \\ Nuclear Medicine, Mayo Clinic, \\ Jacksonville, FL, USA
}

\begin{abstract}
Peptide receptor radionuclide therapy (PRRT) is a paradigm shifting approach to the treatment of neuroendocrine tumors. Although there are no prospective randomized trials directly studying PRRT in pancreatic neuroendocrine tumors (panNETs), there are data to suggest benefit in this patient population. Collectively, the data, consisting of two prospective and six retrospective studies, show a median PFS ranging from 20 to 39 months and a median OS ranging from 37 to 79 months. There are ongoing (and upcoming) prospective, randomized trials of PRRT in panNETs, which will provide further evidence to support this approach.
\end{abstract}

Keywords: pancreatic neuroendocrine tumor, panNET, peptide receptor radionuclide therapy, PRRT, theranostics

\section{Introduction}

Effective therapies for gastroenteropancreatic neuroendocrine tumors (GEP-NETs) beyond first-line somatostatin receptor analogs (SSA) are limited. The introduction of peptide receptor radionuclide therapy (PRRT) has introduced a transformative treatment for patients with GEP-NETs. Approval of PRRT for GEP-NETs in the United States and Europe was primarily based on the NETTER-1 trial. ${ }^{1}$ It is important to point out that the NETTER-1 trial enrolled patients with small bowel NETs only and did not include pancreatic neuroendocrine tumors (panNETs). To date, no randomized, prospective trials of PRRT in patients with panNETs have been reported but such trials are underway. Most of the evidence supporting PRRT for treating patients with panNETs derives from retrospective studies and small prospective Phase II trials. ${ }^{2}$ This review is designed to highlight data for which PRRT has been used in panNETs.

\section{Pancreatic Neuroendocrine Tumors Biology}

PanNETs belong to the diverse group of neuroendocrine neoplasms (NENs) that arise from neuroendocrine cells in the gastrointestinal tract. ${ }^{3}$ Embryologically these tumors are of foregut origin and likely stem from specialized cells in the pancreas referred to as islet cells. ${ }^{4}$ PanNETs are classified based on their grade (grade 1-3), morphologic differentiation (well differentiated vs. poorly differentiated), as well as whether the tumor is functional or non-functional. ${ }^{5}$ Functional tumors can secrete hormones such as insulin, gastrin, glucagon, vasoactive intestinal peptide (VIP), and somatostatin. ${ }^{6}$ As a result of
Correspondence: Jason S Starr

Mayo Clinic, 4500 San Pablo Road,

Jacksonville, FL 32224, USA

Email Starr.Jason@mayo.edu 
excess hormone secretion, panNETs can be associated with a number of syndromes. The most common of these syndromes include insulinoma and Zollinger-Ellison syndrome (gastrin), while the less common include VIPoma (vasoactive intestinal peptide), glucagonoma, and somatostatinoma. The majority of panNETS are nonfunctional. ${ }^{7-9}$

PanNET grading is based on the proliferative rate of the tumor, which is determined based on $\mathrm{Ki}-67$ and/or mitotic index. The latest installment of the WHO Classification of Tumours of Endocrine Organs updated panNETs to include a well-differentiated grade 3 subtype. ${ }^{5}$ Additionally, the cut-off of $\mathrm{Ki}-67$ was changed from $<2 \%$ to $<3 \%$ for grade 1 tumors. The grade of the tumor is helpful for determining prognosis and treatment approach, though clinical behavior and demonstrated pace of growth are important factors as well.

The genomic landscape of this disease has been investigated with next-generation sequencing. ${ }^{10-12}$ Recurring somatic mutations were found to be predominantly in three molecular domains including chromatin remodeling factors (CRFs), histone methyltransferases (HMTs), and genes involved in the mTOR pathway. ${ }^{13}$ The most common altered genes in one study were $M E N 1, D A X X, A T R X$, and TSC2. Epigenetics has been another area of active investigation to help understand pathogenesis and predict the prognosis of panNETs. ${ }^{10,14}$

\section{Epidemiology}

PanNETs are considered rare tumors with an incidence of $<1$ case per 100,000 individuals per year and represent $1 \%$ to $2 \%$ of all pancreatic neoplasms. ${ }^{15}$ The incidence of these tumors has been increasing over the past 40 years from 0.27 to 1.00 per $100,000 .{ }^{16}$ Additionally, patients are increasingly being diagnosed at earlier stages, likely as a result of improved diagnostic methods, namely imaging modalities (ie ${ }^{68} \mathrm{Ga}$ DOTATATE PET/CT, ${ }^{111}$ In pentetreotide) and endoscopic techniques. ${ }^{3}$

The incidence of panNETs is generally comparable between males and females with an incidence of 0.72 and 0.51 per 100,000 patients for males and females, respectively. ${ }^{17}$ The highest incidence of these tumors occurs in the third to sixth decade of life. ${ }^{5}$ Most panNETs are sporadic in nature; however, 10-20\% occur in the setting of an inherited syndrome such as multiple endocrine neoplasia type 1 (MEN1), von Hippel-Lindau (VHL) syndrome, neurofibromatosis type 1 (NF1), and tuberous sclerosis.

\section{Prognosis}

The prognosis of panNETs varies based on stage and grade of the tumor. Tumors $<2 \mathrm{~cm}$ tend to have an excellent prognosis, reflecting an indolent biology/grade. ${ }^{18-21}$ For localized tumors (stage I, II) amenable to resection $>80 \%$ are cured by surgery alone. $^{22}$ For advanced (ie stage IV) disease, there has been significant improvement in the survival of grade 1 and grade 2 panNETs over the past three decades with an improvement of median overall survival from approximately 2 years to 5 years. ${ }^{16}$ Advanced grade 3 panNETs have a less favorable prognosis, although much better than poorly differentiated (grade 3) pancreatic neuroendocrine carcinomas, with 5-year survival rates of $29 \% .{ }^{23}$ At present, there are several molecular markers of interest that may improve prognostication following surgery, most notably mutations in ATRX and DAXX and alternating lengthening of telomeres. ${ }^{4,24-27}$

\section{Treatment}

The treatment of panNETs depends on symptom severity and etiology along with biology and tempo of disease. First-line treatment is typically determined by symptoms, disease bulk, pace of growth, along with disease distribution; specifically liver predominant disease versus diffuse disease (Figure 1). For liver predominant disease, a surgical debulking can be considered (typically if $>70 \%$ of the disease can be removed) and/or liver directed therapies (eg radioembolization, bland embolization, chemoembolization) can be considered. ${ }^{28}$ These interventions can be very helpful for symptom control as well. It is worth mentioning in the era of PRRT that there are limited data for the sequential use of PRRT and radioembolization $\left({ }^{90} \mathrm{Y}\right)$. A study of 20 patients who received sequential PRRT (45\% of whom received this first) and radioembolization showed no increased liver toxicity with either modality in either sequence. ${ }^{29}$ Another study analyzed 23 patients who received radioembolization after PRRT and also saw no increased hepatotoxicity. ${ }^{30}$ Despite this limited data, suggesting this is a safe approach; caution should be exhibited when using these modalities sequentially until further data are available.

For significant extra-hepatic disease, first-line treatments with somatostatin analogues (SSAs) (ie octreotide LAR, lanreotide) are used to treat hormonal syndrome and halt disease progression. ${ }^{31}$ For more bulky, symptomatic disease, capecitabine with temozolomide may be beneficial. ${ }^{32-34}$ The recent ECOG2211 was a prospective, randomized phase II trial that looked at temozolomide (TEM) vs capecitabine and temozolomide (CAPTEM) in advanced well-differentiated grade 1/ 


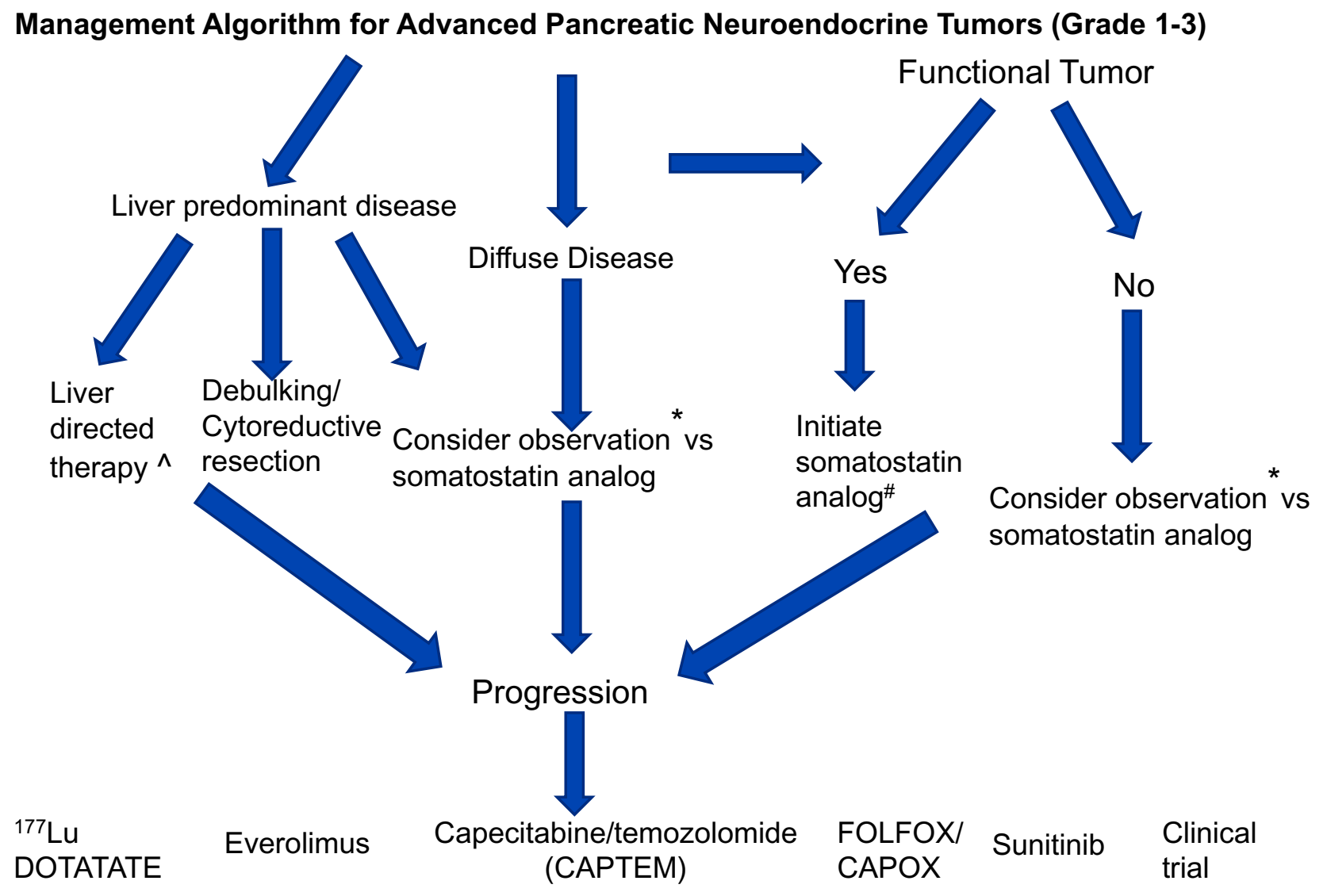

Figure I Algorithm for treatment of advanced panNETs.

grade 2 panNETs. CAPTEM when compared to single-agent TEM improved progression-free survival (PFS) 22.7 months (mo) vs $14.4 \mathrm{mo}(\mathrm{p}=0.023)$, overall survival (OS) not reached vs $38 \mathrm{mo}(\mathrm{p}=0.012)$, and overall response rate (ORR) $33.3 \%$ vs $27.8 \%(p=0.47)$, respectively. Other therapies that can be considered for advanced panNETs include sunitinib and everolimus, both which have been shown to prolong PFS compared to placebo, but ORR is $<10 \%$ with these agents. ${ }^{35,36}$ In January 2018, the peptide receptor radionuclide therapy (PRRT) Lutetium-177 Dotatate (Lutathera ${ }^{\circledR}$ ) was FDA approved for the treatment of advanced somatostatin positive GEP-NETs. This was based on data from the prospective NETTER-1 trial in small bowel NETs and the retrospective Rotterdam, Netherlands experience. ${ }^{1,37}$ It should be noted that NETTER-1 did not include panNETs, and the Netherlands experience at Erasmus included approximately 30\% (91/310) panNETs in its phase II study. ${ }^{37}$ NETTER-1 was a Phase III, prospective, randomized controlled trial that evaluated $177 \mathrm{Lu}-$ DOTATATE combined with $30 \mathrm{mg}$ long-acting repeatable (LAR) versus $60 \mathrm{mg}$ octreotide LAR after first-line progression on SSA alone. 177Lu-DOTATATE as compared to octreotide LAR showed improvements in PFS 28.5 mo vs $8.5 \mathrm{mo}$, and ORR $18 \%$ vs $3 \%$, respectively. ${ }^{38}$ The OS data is still maturing.

\section{Role of Somatostatin Analogues}

Central to identifying somatostatin as a potential target in GEPNETs was the discovery by Jean Claude Reubi (Sandoz Research Institute), Steven Lamberts (Erasmus Medical Center), and Larry Kvols (Mayo Clinic) of the presence of somatostatin receptors in the gastrointestinal tract in $1985 .{ }^{39}$ Somatostatin is a naturally occurring hormone in the body with the highest concentrations in the gastrointestinal tract, pancreas, and central nervous system (especially the pituitary gland). ${ }^{40,41}$ It normally acts as an inhibitory hormone, specifically regulating the release of gastrin, insulin, glucagon, pancreatic amylase, cholecystokinin, among others. ${ }^{42-45}$ There are five different naturally occurring somatostatin receptors (SSTR 1-5) on neuroendocrine cells. SSTR2 has the highest expression in GEP-NETS, at approximately 80-90\%, making this an attractive target. ${ }^{46}$ Based on this finding the SSAs were developed to have the highest affinity for SSTR2. ${ }^{46}$ 
It has been known for many decades that SSAs decrease the neuropeptide/hormone overproduction, which accounts for carcinoid syndrome and cause a similar reduction in the production of hormones resulting in symptomatic improvement that has been seen in patients with functional panNETs. ${ }^{47-49}$ In addition, somatostatin has inhibitory effects on cell proliferation which was further proven clinically with the CLARINET (lanreotide) and PROMID (octreotide long-acting release [LAR]) studies. In the CLARINET study, lanreotide vs placebo yielded a PFS of 32.8 months vs 18 months (95\% CI, 30.9-68), respectively. ${ }^{31}$ (PMID 2674312). The PROMID study, which notably excluded patients with panNETs, found a median PFS of 14.3 mo vs 6 mo in the octreotide LAR and placebo groups, respectively. ${ }^{31,50}$

\section{Biology of PRRT}

The utility of PRRT in NETs rests on the biologic basis of somatostatin receptor expression on the surface of NETs. PRRT consists of a radionuclide (ie Lutetium-177 [ $\left.{ }^{177} \mathrm{Lu}\right]$, Yttrium-90 $\left[{ }^{90} \mathrm{Y}\right]$ ) bound to a chelator (ie DOTA) which is attached to an SSTR ligand, such as $\left[\mathrm{Tyr}^{3}\right]$ octreotate or $\left[\mathrm{Tyr}^{3}\right]$ octreotide. ${ }^{51}$ The compound is given intravenously and the ligand (ie $\left[\mathrm{Tyr}^{3}\right]$ octreotate) binds to the SSTR on the cell surface and subsequently delivers $\beta^{-}$radiation emission. The $\beta^{-}$emission has a range of $2 \mathrm{~mm}$ for ${ }^{177} \mathrm{Lu}$ and $12 \mathrm{~mm}$ for ${ }^{90} \mathrm{Y}$. Of note, ${ }^{177} \mathrm{Lu}$ also emits low-energy $\gamma$ rays which may be of value in scintigraphic confirmation of dose delivery immediately after administration. Furthermore, there is an interest in serial imaging after therapy to calculate a residence time of radiotracer, which may in turn reflect treatment efficacy. ${ }^{52}$ Of the studied compounds, there has been the most clinical experience with ${ }^{177} \mathrm{Lu}-$ DOTATATE and ${ }^{90}$ Y-DOTATOC.

\section{Historical Perspective of PRRT (Figure 2)}

With the development of the first synthetic somatostatin compound, octreotide (Bauer Life Science, Marchbach Pharm Biotechnology,1998), there were soon attempts to couple this compound with a radioisotope, namely iodine $\left[{ }^{123} \mathrm{I}\right]$, because of early success with using this radioisotope in the treatment of thyroid disease. ${ }^{39}$ The use of this compound gave us the first look at the potential of somatostatin expression as a theranostic. In 1990 Eric Krenning and colleagues developed ${ }^{111}$ In-pentetreotide somatostatin scintigraphy (Octreoscan). Based on their experience with this imaging, the FDA approved (in 1994) ${ }^{111}$ In-pentetreotide for the diagnostic imaging of GEP-NETs. ${ }^{53}$ Again, mirroring the experience in thyroid cancer with radioactive iodine, Krenning and his team were able to treat a patient with high doses of ${ }^{111}$ In-pentetreotide; thus successfully delivering the first PRRT treatment in NETs. ${ }^{54}$ This early work set the stage for the later development of more sensitive diagnostic imaging with ${ }^{55} \mathrm{Ga}$-DOTATATE and ultimately identification of the radiopharmaceutical ${ }^{177} \mathrm{Lu}$-DOTATATE. Studies with ${ }^{177}$ Lu-DOTATATE started in 2000 in Rotterdam, Netherlands and led to the international Phase 3 trial, NETTER-1.

\section{PRRT in Pancreatic Neuroendocrine Tumors Efficacy ${ }^{177}$ Lu-DOTATATE}

It should be noted that there have been no randomized, prospective, Phase III trials utilizing PRRT in panNETs. Furthermore, the NETTER-1 trial which is the largest study utilizing PRRT did not include panNETs. However, there are data, both prospective and retrospective, examining the use of PRRT in panNETs. ${ }^{2}$ The median disease control rate (DCR) was $83 \%$ (range, 50-94\%) and the median objective response rate (ORR) was $58 \%$ (range, $13-73 \%$ ). ${ }^{37,56-63}$ The median PFS ranged from 25 to 34 months with a median OS of 42 to 71 months. ${ }^{30,37,57,60,61,64}$

A retrospective study of 74 patients with GEP NET showed that panNET patients had a higher ORR (modified SWOG criteria), $73 \%$ vs $39 \%(\mathrm{p}=0.005)$. There was also a suggestion of longer median OS in panNET (57 vs 45 months); however, this was only noted univariate analysis $(\mathrm{p}=0.037)$ and not multivariate analysis $(\mathrm{p}=0.173) .{ }^{61} \mathrm{In}$ a study of 310 patients with GEP-NETs, the retrospective analysis revealed that those with functional panNETs had reduced disease-specific survival as compared to nonfunctional GEP-NETs, 33 months vs $>48$ months $(\mathrm{p}=0.04)$, respectively. ${ }^{57}$ This was further supported by another retrospective study of 68 patients which showed that on univariate analysis functional panNETs had worse median OS than nonfunctional panNETs, 45 months vs 63 months $(\mathrm{p}=0.045)$, respectively; however, this finding did not hold statistical significance on multivariate analysis $(\mathrm{p}=0.506){ }^{59}$

This study also noted that the grade of the panNET predicted outcomes with PRRT. Grade 1 tumors had a median PFS of 45 months $(95 \%$, CI $35-55)$ as compared to 28 months $(95 \%$, CI $20-36)$ in patients with grade 2 tumors $(\mathrm{p}=0.04)$. In 


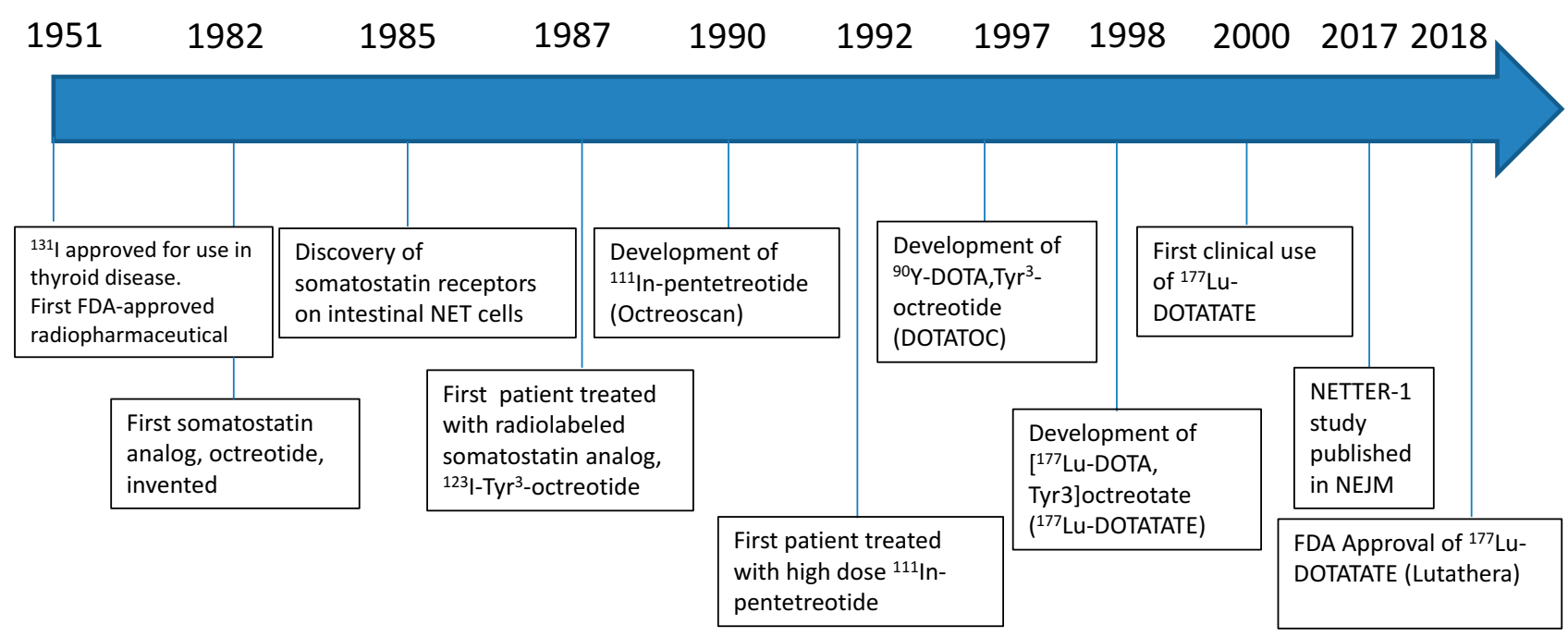

Figure 2 Historical evolution of peptide receptor radionuclide therapy.

addition to functionality and grade of the panNET, the presence of fluorodeoxyglucose (FDG) uptake on positron emission tomography (PET) was noted to be a prognostic factor. ${ }^{64}$ A retrospective analysis of 60 patients identified that those with positive FDG PET had a median PFS of 21.1 months versus 68.7 months in those with a negative FDG PET $(p<0.0002)$. This was also confirmed on multivariate analysis showing an HR of $5.15(95 \%$ CI, $1.42-18.75 ; \mathrm{p}=0.13)$ risk for progression with a positive FDG PET.

\section{${ }^{90} \mathrm{Y}$-DOTATOC and ${ }^{90} \mathrm{Y}$-DOTATATE}

The largest experience utilizing ${ }^{90}$ Y-DOTATOC was in a prospective phase II trial evaluating 342 patients with panNETs (functional, $\mathrm{n}=47$; nonfunctional, $\mathrm{n}=295$ ). Almost half of the patients with panNETs (ORR $=47 \%$, RECIST criteria) experienced tumor response. The reported mean OS in the nonfunctional panNET group was 60 months. ${ }^{65}$ The largest experience with ${ }^{90} \mathrm{Y}$-DOTATATE in panNETs comes from a Phase 2 trial evaluating 30 patients. ${ }^{66}$ In this group, the ORR was 39\% with a median PFS and OS of 25 mo and 42 mo, respectively.

\section{Pooled Analyses of PRRT in panNETs}

Collectively, a total of eight studies ( 2 prospective, 6 retrospective) reported outcomes of PRRT in panNETs (Table 1)..$^{5,67-73}$ The reported median PFS ranged from 20 to 39 months and median OS ranged from 37 to 79 months. Of note, no significant difference in PFS or OS was found when comparing panNETs and other sites of NETs. ${ }^{71,72}$ It should also be noted that these studies are quite heterogeneous in terms of previous lines of therapy administered as well as whether patients had progressive disease when treated.

\section{Functional panNETs}

Two studies evaluated PRRT for treatment of gastrinoma. ${ }^{69,74}$ One of those studies evaluated 11 patients with gastrinoma and noted that all of the patients improved symptomatically; however, the mean survival was only 14 months. ${ }^{74}$ The other study evaluated 36 patients with gastrinoma and showed an ORR of $30 \%$ along with a clinical response of $16 \% .{ }^{69}$ For those deemed responders, the median OS was 45 months. For malignant insulinomas, there is limited data in the form of case reports/series suggesting a benefit of PRRT in both stabilizing disease and hypoglycemia. ${ }^{75,76}$ A recent retrospective study of 34 patients with metastatic functional pNETs and refractory hormonal symptoms found that the majority (71\%) had a significant improvement in the functional syndrome and $80 \%$ had a reduction in the corresponding circulating hormone levels. After PRRT, the median PFS was 18.1 months and was associated with a concurrent increase in quality of life (QoL). ${ }^{77}$ A similar but smaller study of 11 patients with refractory secretory symptoms was recently reported and most patients experienced symptomatic improvement following PRRT. ${ }^{78}$ As an example, 4 of 5 patients with insulinoma and refractory hyperglycemia improved. Among the patients with symptomatic gastrinoma and glucagonoma, symptomatic improvement was not as consistent. 
Table I Efficacy of PRRT in panNET

\begin{tabular}{|c|c|c|c|c|c|c|c|}
\hline Study & Radiopharmaceutical & Study Type & $\begin{array}{l}\text { No. of } \\
\text { panNET } \\
\text { Patients }\end{array}$ & $\begin{array}{l}\text { Response } \\
\text { Criteria }\end{array}$ & ORR & $\begin{array}{l}\text { mPFS } \\
\text { Months } \\
(95 \% \mathrm{Cl})\end{array}$ & $\begin{array}{l}\text { mOS } \\
\text { Months } \\
(95 \% \mathrm{Cl})\end{array}$ \\
\hline Baum 55 & $\begin{array}{l}{ }^{177} \text { Lu-PRRT }(36 \%),{ }^{90} \text { Y-PRRT (15\%) or both } \\
(49 \%)\end{array}$ & Retrospective & 384 & RECIST & NS & $20(17-23)$ & $44(38-50)$ \\
\hline Sharma $^{73}$ & ${ }^{90}$ Y-PRRT $(83 \%),{ }^{177}$ Lu-PRRT (I5\%) & Retrospective & 35 & Non-standard & NS & NS & $37(18-48)$ \\
\hline Kunikowska $^{72}$ & ${ }^{90}$ Y-DOTATATE \& ${ }^{177}$ Lu-DOTATATE & Prospective & 19 & RECIST & NS & 30 & 79 \\
\hline Bertani $^{71}$ & $\begin{array}{l}{ }^{90} \text { Y-DOTATOC }(37 \%),{ }^{177} \text { Lu-DOTATATE } \\
(28 \%) \text {, both }(35 \%)\end{array}$ & Prospective & 90 & RECIST & $26 \%$ & $36(24-44)$ & $75(64-104)$ \\
\hline Horsch $^{70}$ & $\begin{array}{l}{ }^{177} \text { Lu-PRRT (54\%), }{ }^{90} \text { Y-PRRT (I7\%), both } \\
(29 \%)\end{array}$ & Retrospective & 172 & RECIST & NS & 39 (29-49) & $53(37-69)$ \\
\hline Dumont $^{69}$ & $\begin{array}{l}{ }^{90} \text { Y-DOTATOC (80\%) or }{ }^{90} \text { Y-DOTATOC \& } \\
{ }^{177} \text { Lu-DOTATOC }(20 \%)\end{array}$ & Prospective & 36 & NS & $33 \%$ & NS & 40 \\
\hline Campana $^{68}$ & ${ }^{90}$ Y-DOTATOC or ${ }^{177}$ Lu-DOTATATE & Retrospective & 45 & RECIST & $31 \%$ & 23 & NS \\
\hline Pfeifer ${ }^{67}$ & $\begin{array}{l}{ }^{90} \text { Y-DOTATOC (77\%), }{ }^{177} \text { Lu-DOTATOC or } \\
\text { both }(23 \%)\end{array}$ & Retrospective & 21 & RECIST & $33 \%$ & 27 & NR \\
\hline
\end{tabular}

Abbreviations: PRRT, peptide receptor radionuclide therapy; panNET, pancreatic neuroendocrine tumor; ORR, overall response rate; mPFS, median progression-free survival; mOS, median overall survival; NS, not stated; NR, not reached; $\mathrm{Cl}$, confidence interval.

\section{Quality of Life (QoL) Measures}

Very few studies have reported QoL measures in patients with panNET being treated with PRRT. The largest such study is a retrospective study in 68 patients with advanced panNET. ${ }^{79}$ QoL was evaluated with the European Organization for Research and Treatment of Cancer - Quality of Life Questionnaire for Cancer patients, 30 items. Patients who received four cycles of PRRT did not have an adverse impairment of QoL in any measure. After 3 months from the last cycle of PRRT, it was noted that patients had significant improvement in global health status, social functioning, and symptoms of fatigue and appetite loss. These findings are consistent with a recent update regarding health-related QoL outcomes from the NETTER-1 trial as well as from the Erasmus group. ${ }^{77,80}$

\section{Safety}

Overall, PRRT is a safe treatment and most adverse effects are transient. The nausea frequently seen following administration of the drugs has decreased substantially among patients receiving compounded nephroprotective arginine and lysine amino acid infusion as compared to commercially available amino acid solutions with more amino acids than arginine and lysine. Nephrotoxicity seems to be most frequently associated with PRRT using ${ }^{90} \mathrm{Y}$ and is virtually nonexistent when ${ }^{177} \mathrm{Lu}$ PRRT is given with nephroprotective amino acid infusion. Pooled analysis by Ramage et al reported on six studies that analyzed hematologic adverse events. ${ }^{2}$ Two retrospective studies utilizing
${ }^{177} \mathrm{Lu}$-DOTATATE revealed a hematologic grade $3 / 4 \mathrm{AE}$ rate of $5-7 \%$, while the other four studies had no grade $3 / 4$ hematologic adverse events. ${ }^{59,64,69,81-83}$ This compares to a rate of $1-9 \%$ grade $3 / 4$ hematologic AEs (mostly lymphopenia) in the NETTER-1. ${ }^{1}$ In addition, permanent hematologic dysfunction has been reported in multiple studies with therapy-related myeloid neoplasms (t-MN) and bone marrow failures. ${ }^{1,55,84-87}$ However, the incidence rates along with the clinical features of t-MN are variable across studies, ranging between $2 \%$ and $5 \%$. ${ }^{1,37,57,60,65,72,84,87-104}$ However, the majority of the studies have significant bias (selection bias, publication bias) due to their retrospective nature. Of note, the t-MN incidence in the PRRT group in the NETTER-1 trial was $0.9 \%{ }^{1}$ However, the period of follow-up was short to accurately determine such risk. Prior studies have evaluated potential risk factors for developing t-MN after PRRT such as prior alkylating agents, metastatic disease to the bone, prior radiation, and others. ${ }^{84,87,90}$ However, these factors have not been consistently implicated across studies.

\section{Future Directions}

There is much work to be done with the future application of PRRT in panNETs. First, it is worth mentioning the soon to be opened NETTER-2 trial (NCT03972488) which is a phase 3 , randomized, study with ${ }^{177} \mathrm{Lu}$-DOTATATE with $30 \mathrm{mg}$ octreotide LAR versus $60 \mathrm{mg}$ octreotide LAR, in the first-line treatment of grade 2 and grade 3 advanced GEP-NETs. 
Another study of interest is the COMPETE trial (NCT03049189) which is another phase 3, randomized, trial studying PRRT $\left({ }^{177}\right.$ Lu-DOTATOC) vs everolimus in the first-line treatment of advanced GEP-NETs (all grades included). Collectively the aforementioned somatostatin analogues, PRRT included, work in an agnostic fashion in binding of SSTR (namely SSTR2). Alternatively, there is a body of evidence that suggests that somatostatin antagonists, although not internalized into the cell, have improved binding capacity to SSTR and thus can potentially deliver higher doses of radiation to the tumor and provide improved theranostic-based imaging. ${ }^{105-107}$ One theranostic grouping studied in clinical trials (NCT03773133, NCT 02592707) has two somatostatin antagonists, one for imaging, ${ }^{55} \mathrm{Ga}-$ OPS202, and the other as the companion PRRT agent, ${ }^{177} \mathrm{Lu}-$ OPS201 (satoreotide).

Other potential strategies of interest are combining cytotoxic chemotherapy with PRRT. Investigators in Australia looked at 30 patients with grade 1 or 2 panNET and combined PRRT (4 doses of ${ }^{177}$ Lu-Dotatate) with CAPTEM (14 days of chemotherapy every 8 weeks during PRRT). This yielded an ORR of $80 \%$ with a median PFS of 48 months. ${ }^{108}$ In Australia, the majority of patients receive 5-FU radiosensitizing chemotherapy with PRRT treatment. ${ }^{109}$ A recent retrospective analysis from Australia revealed an incidence of t-MN as high as $4.8 \%$, with $90 \%$ of the patients receiving chemotherapy with PRRT. ${ }^{85}$ This highlights a word of caution regarding combining chemotherapy with PRRT and certainly more data are needed with this approach before being routinely employed.

In the era of immunotherapy, it is easy to foresee an approach that combines PRRT with immunotherapy (ie checkpoint inhibitors). Radiotherapy has been shown to increase tumor antigenicity as well as increase antigen presentation which in turn can enhance T-cell destruction of tumor cells. ${ }^{110}$ Preclinical data of a NET xenograft model treated with PRRT showed increased infiltration of antigen-presenting cells and NK cells in the tumor microenvironment. ${ }^{111}$ Studies employing a strategy of PRRT and immunotherapy are in development.

There are also questions that remain in terms of sequencing of therapy for panNETs. We have the ECOG2211 first-line data utilizing CAPTEM that showed impressive results in the treatment of this disease and it is unclear whether PRRT should be considered before or after. Additionally, as alluded to above it is unclear whether the sequencing of therapies increases the risk of t-MN. A trial looking at the sequencing of therapies would be important to help answer this question.
Another frontier that is being explored as it relates to PRRT is the use of alpha $(\alpha)$ particles, namely ${ }^{225}$ Actinium- and ${ }^{213}$ Bismuth-DOTATOC therapy, as the radiation source. ${ }^{112}$ The advantages of $\alpha$ particle therapy include that they deliver radiation over a short range of emission $(<0.1 \mathrm{~mm})$ which helps spare damage to surrounding normal tissue. ${ }^{113}$ Additionally, alpha radiation delivers a higher linear energy transfer than beta $(\beta)$ particles, thus can be more effective at inducing DNA doublestrand breaks. Another such agent is ${ }^{212} \mathrm{~Pb}$-DOTAMTATE completed a Phase I study with plans to open phase II in the near future. Alpha particle PRRT has the potential to usher in a new era in radiopharmaceuticals.

\section{Conclusion}

While many therapies in the oncology as of late offer small, incremental benefits; PRRT has represented a true breakthrough in the treatment of NETs. Although prospective, randomized data with PRRT in panNETs are lacking, the data that we do have suggest this is an effective treatment for this disease, and absolutely warrants further investigation. Future efforts will be directed at enrolling patients on the clinical trials mentioned in this review (COMPETE and NETTER-2 trials) so that the medical community has stronger data to make treatment recommendations in panNET. Equally important will be the determination of sequencing of PRRT with the other therapies available, namely chemotherapy and immunotherapy. It will also be paramount to continue to assess long-term risks of PRRT, specifically the incidence of t-MN. Considering the caveats of the current data, PRRT represents an effective and promising therapy in the treatment of panNETs.

\section{Disclosure}

Dr Timothy Hobday reports personal fees from Lexicon Pharmaceuticals, outside the submitted work. Dr Ayse Tuba Kendi was the principal investigator of Phase III VISION study in the treatment of metastatic castrationresistant prostate cancer patients with Lu-PSMA-617, at Mayo Clinic, outside the submitted work. Dr Thorvardur Halfdanarson reports grants that paid to the institution from Ipsen and Thermo Fisher Scientific; personal fees from Curium and Lexicon, during the conduct of the study. The authors report no other conflicts of interest in this work. 


\section{References}

1. Strosberg J, El-Haddad G, Wolin E, et al. Phase 3 trial of 177Lu-dotatate for midgut neuroendocrine tumors. $N$ Engl J Med. 2017;376(2):125-135. doi:10.1056/NEJMoa1607427

2. Ramage J, Naraev BG, Halfdanarson TR. Peptide receptor radionuclide therapy for patients with advanced pancreatic neuroendocrine tumors. Semin Oncol. 2018;45(4):236-248. doi:10.1053/j. seminoncol.2018.08.004

3. Alsidawi S, Westin GFM, Hobday TJ, Halfdanarson TR. Pancreatic neuroendocrine tumors: a population-based analysis of epidemiology and outcomes. J Clin Oncol. 2017;35 (4_suppl):401. doi:10.1200/JCO.2017.35.4_suppl.401

4. Chan CS, Laddha SV, Lewis PW, et al. ATRX, DAXX or MEN1 mutant pancreatic neuroendocrine tumors are a distinct alpha-cell signature subgroup. Nat Commun. 2018;9(1):4158. doi:10.1038/ s41467-018-06498-2

5. Lloyd RV, Osamura RY, Klöppel G, Rosai J. WHO Classification of Tumours of Endocrine Organs. WHO Classification of Tumours. 4th ed., Vol. 10. Lyon, France: International Agency for Research on Cancer (IARC); 2017.

6. Halfdanarson TR, Rubin J, Farnell MB, Grant CS, Petersen GM. Pancreatic endocrine neoplasms: epidemiology and prognosis of pancreatic endocrine tumors. Endocr Relat Cancer. 2008;15 (2):409-427. doi:10.1677/ERC-07-0221

7. Kasumova GG, Tabatabaie O, Eskander MF, et al. National rise of primary pancreatic carcinoid tumors: comparison to functional and nonfunctional pancreatic neuroendocrine tumors. $\mathrm{J}$ Am Coll Surg. 2017;224(6):1057-1064. doi:10.1016/j.jamcollsurg.2016.11.014

8. Halfdanarson TR, Rabe KG, Rubin J, Petersen GM. Pancreatic neuroendocrine tumors (PNETs): incidence, prognosis and recent trend toward improved survival. Ann Oncol. 2008;19 (10):1727-1733. doi:10.1093/annonc/mdn351

9. Zerbi A, Falconi M, Rindi G, et al. Clinicopathological features of pancreatic endocrine tumors: a prospective multicenter study in Italy of 297 sporadic cases. Am J Gastroenterol. 2010;105 (6):1421-1429. doi:10.1038/ajg.2009.747

10. Mafficini A, Scarpa A. Genetics and epigenetics of gastroenteropancreatic neuroendocrine neoplasms. Endocr Rev. 2019;40 (2):506-536. doi:10.1210/er.2018-00160

11. Mafficini A, Scarpa A. Genomic landscape of pancreatic neuroendocrine tumours: the International Cancer Genome Consortium. $J$ Endocrinol. 2018;236(3):R161-R167. doi:10.1 530/JOE-17-0560

12. Scarpa A, Chang DK, Nones K, et al. Whole-genome landscape of pancreatic neuroendocrine tumours. Nature. 2017;543 (7643):65-71. doi:10.1038/nature21063

13. Raj N, Shah R, Stadler Z, et al. Real-time genomic characterization of metastatic pancreatic neuroendocrine tumors has prognostic implications and identifies potential germline actionability. JCO Precis Oncol. 2018;2018. doi:10.1200/PO.17.00267

14. Pipinikas CP, Dibra H, Karpathakis A, et al. Epigenetic dysregulation and poorer prognosis in DAXX-deficient pancreatic neuroendocrine tumours. Endocr Relat Cancer. 2015;22(3):L13-L18. doi:10.1530/ERC-15-0108

15. Hallet J, Law CHL, Cukier M, et al. Exploring the rising incidence of neuroendocrine tumors: a population-based analysis of epidemiology, metastatic presentation, and outcomes. Cancer. 2015;121(4):589-597. doi:10.1002/cncr.29099

16. Dasari A, Shen C, Halperin D, et al. Trends in the incidence, prevalence, and survival outcomes in patients with neuroendocrine tumors in the United States. JAMA Oncol. 2017;3 (10):1335-1342. doi:10.1001/jamaoncol.2017.0589

17. Sonbol MB, G M, Starr J, Hobday T, Halfdanarson T. 2019 Annual North American Neuroendocrine Tumor Society (NANETS) Meeting; Boston, USA; 2019.
18. Marchegiani G, Landoni L, Andrianello S, et al. Patterns of recurrence after resection for pancreatic neuroendocrine tumors: who, when, and where? Neuroendocrinology. 2018. doi:10.1159/ 000495774

19. Teo RYA, Teo TZ, Tai DWM et al. Systematic review of current prognostication systems for pancreatic neuroendocrine neoplasms. Surgery. 2019;165(4):672-685. doi:10.1016/j.surg.2018.10.031

20. Landoni L, Marchegiani G, Pollini T, et al. The evolution of surgical strategies for Pancreatic Neuroendocrine Tumors (Pan-NENs): time-trend and outcome analysis from 587 consecutive resections at a high-volume institution. Ann Surg. 2019;269 (4):725-732. doi:10.1097/SLA.0000000000002594

21. Sallinen VJ, Le Large TYS, Tieftrunk E, et al. Prognosis of sporadic resected small $(\leq 2 \mathrm{~cm})$ nonfunctional pancreatic neuroendocrine tumors - a multi-institutional study.. HPB (Oxford). 2018;20(3):251-259. doi:10.1016/j.hpb.2017.08.034

22. Howlader NNA, Krapcho M, et al. SEER cancer statistics review, 1975-2016. 2018.

23. Basturk O, Yang Z, Tang LH, et al. The high-grade (WHO G3) pancreatic neuroendocrine tumor category is morphologically and biologically heterogenous and includes both well differentiated and poorly differentiated neoplasms. Am J Surg Pathol. 2015;39 (5):683-690. doi:10.1097/PAS.0000000000000408

24. Park JK, Paik WH, Lee K, et al. DAXX/ATRX and MEN1 genes are strong prognostic markers in pancreatic neuroendocrine tumors. Oncotarget. 2017;8(30):49796-49806. doi:10.18632/ oncotarget.17964

25. Pea A, Yu J, Marchionni L, et al. Genetic analysis of small well-differentiated pancreatic neuroendocrine tumors identifies subgroups with differing risks of liver metastases. Ann Surg. 2018. doi:10.1097/SLA.000000000003022

26. Marinoni I, Kurrer AS, Vassella E, et al. Loss of DAXX and ATRX are associated with chromosome instability and reduced survival of patients with pancreatic neuroendocrine tumors. Gastroenterology. 2014;146(2):453-460. doi:10.1053/j.gastro.2013.10.020

27. Jiao Y, Shi C, Edil BH, et al. DAXX/ATRX, MEN1, and mTOR pathway genes are frequently altered in pancreatic neuroendocrine tumors. Science. 2011;331(6021):1199-1203. doi:10.1126/ science. 1200609

28. Morgan RE, Pommier SJ, Pommier RF. Expanded criteria for debulking of liver metastasis also apply to pancreatic neuroendocrine tumors. Surgery. 2018;163(1):218-225. doi:10.1016/j. surg.2017.05.030

29. Geisler J, Auernhammer C, Zech C, et al. Toxicity of sequential treatment with radioembolization and PRRT in hepatic metastasized neuroendocrine tumors. J Nucl Med. 2012;53:1189.

30. Ezziddin S, Meyer C, Kahancova S, et al. 90 Y radioembolization after radiation exposure from peptide receptor radionuclide therapy. J Nucl Med. 2012;53(11):1663-1669. doi:10.2967/ jnumed.112.107482

31. Caplin ME, Pavel M, Ćwikła JB, et al. Lanreotide in metastatic enteropancreatic neuroendocrine tumors. N Engl J Med. 2014;371 (3):224-233. doi:10.1056/NEJMoa1316158

32. Kunz PL, Catalano PJ, Nimeiri $\mathrm{H}$, et al. A randomized study of temozolomide or temozolomide and capecitabine in patients with advanced pancreatic neuroendocrine tumors: a trial of the ECOG-ACRIN Cancer Research Group (E2211). J Clin Oncol. 2018;36(suppl; abstr 4004):4004. doi:10.1200/JCO.2018.36.15_suppl.4004

33. Cives M, Ghayouri M, Morse B, et al. Analysis of potential response predictors to capecitabine/temozolomide in metastatic pancreatic neuroendocrine tumors. Endocr Relat Cancer. 2016;23(9):759-767. doi:10.1530/ERC-16-0147

34. Strosberg JR, Fine RL, Choi J, et al. First-line chemotherapy with capecitabine and temozolomide in patients with metastatic pancreatic endocrine carcinomas. Cancer. 2011;117(2):268-275. doi: $10.1002 /$ cncr. 25425 
35. Yao JC, Shah MH, Ito T, et al. Everolimus for advanced pancreatic neuroendocrine tumors. N Engl J Med. 2011;364(6):514-523. doi:10.1056/NEJMoa1009290

36. Raymond E, Dahan L, Raoul J-L, et al. Sunitinib malate for the treatment of pancreatic neuroendocrine tumors. $N$ Engl J Med. 2011;364(6):501-513. doi:10.1056/NEJMoa1003825

37. Brabander T, van der Zwan WA, Teunissen JJM, et al. Long-term efficacy, survival, and safety of [(177)Lu-DOTA(0), Tyr(3)] octreotate in patients with gastroenteropancreatic and bronchial neuroendocrine tumors. Clin Cancer Res. 2017;23 (16):4617-4624. doi:10.1158/1078-0432.CCR-16-2743

38. Strosberg J, Wolin E, Chasen B, et al. First update on overall survival, progression-free survival, and health-related time-todeterioration quality of life from the NETTER-1 study: 177Lu-Dotatate vs. high dose octreotide in progressive midgut neuroendocrine tumors. J Clin Oncol. 2018;36.

39. Levine R, Krenning EP. Clinical history of the theranostic radionuclide approach to neuroendocrine tumors and other types of cancer: historical review based on an interview of Eric P. Krenning by Rachel Levine. J Nucl Med. 2017;58 (Supplement 2):3S-9S. doi:10.2967/jnumed.116.186502

40. Cakir M, Dworakowska D, Grossman A. Somatostatin receptor biology in neuroendocrine and pituitary tumours: part 1 - molecular pathways. J Cell Mol Med. 2010;14(11):2570-2584. doi:10.1111/j.1582-4934.2010.01125.x

41. Dubois MP. Immunoreactive somatostatin is present in discrete cells of the endocrine pancreas. Proc Natl Acad Sci USA. 1975;72(4):1340-1343. doi:10.1073/pnas.72.4.1340

42. Bloom SR, Mortimer CH, Thorner MO, et al. Inhibition of gastrin and gastric-acid secretion by growth-hormone release-inhibiting hormone. Lancet. 1974;304(7889):1106-1109. doi:10.1016/ s0140-6736(74)90869-1

43. Rorsman P, Huising MO. The somatostatin-secreting pancreatic $\delta$-cell in health and disease. Nat Rev Endocrinol. 2018;14 (7):404-414. doi:10.1038/s41574-018-0020-6

44. Mandarino L, Stenner D, Blanchard W, et al. Selective effects of somatostatin-14, -25 and -28 on in vitro insulin and glucagon secretion. Nature. 1981;291(5810):76-77. doi:10.1038/291076a0

45. Ohnishi H, Mine T, Kojima I. Inhibition by somatostatin of amylase secretion induced by calcium and cyclic AMP in rat pancreatic acini. Biochem J. 1994;304(2):531-536. doi:10.1042/bj3040531

46. Öberg K, Kvols L, Caplin M, et al. Consensus report on the use of somatostatin analogs for the management of neuroendocrine tumors of the gastroenteropancreatic system. Ann Oncol. 2004;15(6):966-973. doi:10.1093/annonc/mdh216

47. Strosberg JR, Halfdanarson TR, Bellizzi AM, et al. The North American Neuroendocrine Tumor Society consensus guidelines for surveillance and medical management of midgut neuroendocrine tumors. Pancreas. 2017;46(6):707-714. doi:10.1097/ MPA.0000000000000850

48. Oberg K. Management of functional neuroendocrine tumors of the pancreas. Gland Surg. 2018;7(1):20-27. doi:10.21037/ gs.2017.10.08

49. Raj N, Reidy-Lagunes D. Systemic therapies for advanced pancreatic neuroendocrine tumors. Hematol Oncol Clin North Am. 2016;30(1):119-133. doi:10.1016/j.hoc.2015.09.005

50. Rinke A, Müller -H-H, Schade-Brittinger C, et al. Placebocontrolled, double-blind, prospective, randomized study on the effect of octreotide LAR in the control of tumor growth in patients with metastatic neuroendocrine midgut tumors: a report from the PROMID Study Group. J Clin Oncol. 2009;27 (28):4656-4663. doi:10.1200/JCO.2009.22.8510

51. van der Zwan WA, Bodei L, Mueller-Brand J, et al. GEPNETs update: radionuclide therapy in neuroendocrine tumors. Eur J Endocrinol. 2015;172(1):R1-R8. doi:10.1530/EJE-14-0488
52. Garkavij M, Nickel M, Sjögreen-Gleisner K, et al. 177Lu[DOTA0, Tyr3] octreotate therapy in patients with disseminated neuroendocrine tumors: analysis of dosimetry with impact on future therapeutic strategy. Cancer. 2010;116(S4):1084-1092. doi:10.1002/cncr.24796

53. Lamberts SW, Barker WH, Reubi J-C, Krenning EP. Somatostatin-receptor imaging in the localization of endocrine tumors. N Engl J Med. 1990;323(18):1246-1249. doi:10.1056/ NEJM199011013231805

54. Krenning EP, Kooij PP, Bakker WH, et al. Radiotherapy with a radiolabeled somatostatin analogue, [111In-DTPA-D-Phe1]octreotide. A case history. Ann $N$ Y Acad Sci. 1994;733:496-506. doi:10.1111/j.1749-6632.1994.tb17300.x

55. Baum RP, Kluge AW, Kulkarni H, et al. [(177)Lu-DOTA](0)D-Phe(1)-Tyr(3)-octreotide 177 (177)Lu-DOTATOC) 177 Lu-DOTATOC) for peptide receptor radiotherapy in patients with advanced neuroendocrine tumours: a Phase-II Study. Theranostics. 2016;6(4):501-510. doi:10.7150/thno.13702

56. Kwekkeboom DJ, Bakker WH, Kam BL, et al. Treatment of patients with gastro-entero-pancreatic (GEP) tumours with the novel radiolabelled somatostatin analogue $\left[{ }^{177} \mathrm{Lu}-\mathrm{DOTA}^{0}, \mathrm{Tyr}^{3}\right]$ octreotate. Eur J Nucl Med Mol Imaging. 2003;30(3):417-422. doi:10.1007/s00259-002-1050-8

57. Kwekkeboom DJ, de Herder WW, Kam BL, et al. Treatment with the radiolabeled somatostatin analog [177 Lu-DOTA 0, Tyr 3] Octreotate: toxicity, efficacy, and survival. J Clin Oncol. 2008;26 (13):2124-2130. doi:10.1200/jco.2007.15.2553

58. Bodei L, Cremonesi M, Grana CM, et al. Peptide receptor radionuclide therapy with 177Lu-DOTATATE: the IEO Phase I-II study. Eur J Nucl Med Mol Imaging. 2011;38(12):2125-2135. doi:10.1007/s00259-011-1902-1

59. Ezziddin S, Khalaf F, Vanezi M, et al. Outcome of peptide receptor radionuclide therapy with $177 \mathrm{Lu}$-octreotate in advanced grade 1/2 pancreatic neuroendocrine tumours. Eur J Nucl Med Mol Imaging. 2014;41(5):925-933. doi:10.1007/s00259-0132677-3

60. Garske-Roman U, Sandström M, Fröss Baron K, et al. Prospective observational study of 177Lu-DOTA-octreotate therapy in 200 patients with advanced metastasized neuroendocrine tumours (NETs): feasibility and impact of a dosimetry-guided study protocol on outcome and toxicity. Eur J Nucl Med Mol Imaging. 2018;45(6):970-988. doi:10.1007/s00259-018-3945-Z

61. Ezziddin S, Attassi M, Yong-Hing CJ, et al. Predictors of long-term outcome in patients with well-differentiated gastroenteropancreatic neuroendocrine tumors after peptide receptor radionuclide therapy with $177 \mathrm{Lu}$-octreotate. $J$ Nucl $\mathrm{Med}$. 2014;55(2):183-190. doi:10.2967/jnumed.113.125336

62. Sampaio IL, Luiz HV, Violante LS, et al. Tratamento de Tumores Neuroendócrinos Gastroenteropancreáticos com 177Lu-DOTA-TATE: experiência do Instituto Português de Oncologia do Porto. Acta Médica Portuguesa. 2016;29 (11):726-733. doi:10.20344/amp.7306

63. Demirci E, Kabasakal L, Toklu T, et al. 177Lu-DOTATATE therapy in patients with neuroendocrine tumours including high-grade (WHO G3) neuroendocrine tumours: response to treatment and long-term survival update. Nucl Med Commun. 2018;39 (8):789-796. doi:10.1097/MNM.0000000000000874

64. Sansovini M, Severi S, Ianniello A, et al. Long-term follow-up and role of FDG PET in advanced pancreatic neuroendocrine patients treated with (177)Lu-D OTATATE. Eur J Nucl Med Mol Imaging. 2017;44(3):490-499. doi:10.1007/s00259-016-3533-z

65. Imhof A, Brunner P, Marincek N, et al. Response, survival, and long-term toxicity after therapy with the radiolabeled somatostatin analogue [90 Y-DOTA]-TOC in metastasized neuroendocrine cancers. J Clin Oncol. 2011;29(17):2416-2423. doi:10.1200/ JCO.2010.33.7873 
66. Rogowski W, Wachuła E, Lewczuk A, et al. Long-term efficacy of 90 Y-DOTATATE in patients with nonresectable pancreatic and small bowel neuroendocrine neoplasms. Future Oncol. 2016;12 (16):1877-1885. doi:10.2217/fon-2016-0031

67. Pfeifer AK, Gregersen T, Grønbæk H, et al. Peptide receptor radionuclide therapy with Y-DOTATOC and (177)Lu-DOTATOC in advanced neuroendocrine tumors: results from a Danish cohort treated in Switzerland. Neuroendocrinology. 2011;93:189-196. doi:10.1159/000324096

68. Campana D, Capurso G, Partelli S, et al. Radiolabelled somatostatin analogue treatment in gastroenteropancreatic neuroendocrine tumours: factors associated with response and suggestions for therapeutic sequence. Eur J Nucl Med Mol Imaging. 2013;40 (8):1197-1205. doi:10.1007/s00259-013-2402-2

69. Dumont RA, Seiler D, Marincek N, et al. Survival after somatostatin based radiopeptide therapy with (90)Y-DOTATOC vs. (90) Y-DOTATOC plus (177)Lu-DOTATOC in metastasized gastrinoma. Am J Nucl Med Mol Imaging. 2015;5(1):46-55.

70. Horsch D, Ezziddin S, Haug A, et al. Effectiveness and side-effects of peptide receptor radionuclide therapy for neuroendocrine neoplasms in Germany: a multi-institutional registry study with prospective follow-up. Eur J Cancer. 2016;58:41-51. doi:10.1016/j.ejca.2016.01.009

71. Bertani E, Fazio N, Radice D, et al. Resection of the primary tumor followed by peptide receptor radionuclide therapy as upfront strategy for the treatment of G1-G2 pancreatic neuroendocrine tumors with unresectable liver metastases. Ann Surg Oncol. 2016;23(S5):981-989. doi:10.1245/s10434-016-5550-3

72. Kunikowska J, Pawlak D, Bąk MI, et al. Long-term results and tolerability of tandem peptide receptor radionuclide therapy with (90)Y/(177)Lu-DOTATATE in neuroendocrine tumors with respect to the primary location: a 10-year study. Ann Nucl Med. 2017;31(5):347-356. doi:10.1007/s12149-017-1163-6

73. Sharma N, Naraev BG, Engelman EG, et al. Peptide receptor radionuclide therapy outcomes in a North American cohort with metastatic well-differentiated neuroendocrine tumors. Pancreas. 2017;46(2):151-156. doi:10.1097/MPA.0000000000000734

74. Grozinsky-Glasberg S, Barak D, Fraenkel M, et al. Peptide receptor radioligand therapy is an effective treatment for the long-term stabilization of malignant gastrinomas. Cancer. 2011;117 (7):1377-1385. doi:10.1002/cncr.25646

75. Van Schaik E, van Vliet EI, Feelders RA, et al. Improved control of severe hypoglycemia in patients with malignant insulinomas by peptide receptor radionuclide therapy. J Clin Endocrinol Metab. 2011;96(11):3381-3389. doi:10.1210/jc.2011-1563

76. Magalhaes D, Sampaio IL, Ferreira G, et al. Peptide receptor radionuclide therapy with (177)Lu-DOTA-TATE as a promising treatment of malignant insulinoma: a series of case reports and literature review. J Endocrinol Invest. 2019;42(3):249-260. doi:10.1007/s40618-018-0911-3

77. Zandee WT, Brabander T, Blažević A, et al. Symptomatic and radiological response to $177 \mathrm{Lu}$-DOTATATE for the treatment of functioning pancreatic neuroendocrine tumors. J Clin Endocrinol Metab. 2019;104(4):1336-1344. doi:10.1210/jc.2018-01991

78. Sullivan LAE, Hayes AR, et al. The efficacy of lutetium-177 DOTATATE in refractory symptoms of functional pancreatic neuroendocrine tumours. 2019.

79. Marinova M, Mücke M, Mahlberg L, et al. Improving quality of life in patients with pancreatic neuroendocrine tumor following peptide receptor radionuclide therapy assessed by EORTC QLQ-C30. Eur J Nucl Med Mol Imaging. 2018;45(1):38-46. doi:10.1007/s00259-017-3816-z

80. Strosberg J, Wolin E, Chasen B, et al. Health-related quality of life in patients with progressive midgut neuroendocrine tumors treated with 177 Lu-Dotatate in the Phase III NETTER-1 trial. J Clin Oncol. 2018;36(25):2578-2584. doi:10.1200/ JCO.2018.78.5865
81. Sabet A, Ezziddin K, Hense J, et al. Long-term hematotoxicity after peptide receptor radionuclide therapy with $177 \mathrm{Lu}$-octreotate. Eur J Nucl Med Mol Imaging. 2012;2:S233.

82. Sansovini M, Severi S, Ambrosetti A, et al. Treatment with the radiolabelled somatostatin analog 177 Lu-DOTATATE for advanced pancreatic neuroendocrine tumors. Neuroendocrinology. 2013;97(4):347-354. doi:10.1159/ 000348394

83. Sowa-Staszczak A, Stefanska A, Chrapczynski P, et al. Does combination of "cold" and "hot" somatostatin analogs prolong survival of patients with neuroendocrine neoplasms? Endocr J. 2017;64(2):171-177. doi:10.1507/endocrj.EJ16-0219

84. Bodei L, Kidd M, Paganelli G, et al. Long-term tolerability of PRRT in 807 patients with neuroendocrine tumours: the value and limitations of clinical factors. Eur J Nucl Med Mol Imaging. 2015;42(1):5-19. doi:10.1007/s00259-014-2893-5

85. Goncalves I, Burbury K, Michael M, et al. Characteristics and outcomes of therapy-related myeloid neoplasms after peptide receptor radionuclide/chemoradionuclide therapy (PRRT/ PRCRT) for metastatic neuroendocrine neoplasia: a single-institution series. Eur J Nucl Med Mol Imaging. 2019;46 (9):1902-1910. doi:10.1007/s00259-019-04389-2

86. Kesavan M, Turner JH. Myelotoxicity of peptide receptor radionuclide therapy of neuroendocrine tumors: a decade of experience. Cancer Biother Radiopharm. 2016;31(6):189-198. doi:10.1089/cbr.2016.2035

87. Bergsma H, van Lom K, Raaijmakers MHGP, et al. Persistent hematologic dysfunction after peptide receptor radionuclide therapy with (177)Lu-DOTATATE: 177 Lu-DOTATATE: incidence, course, and predicting factors in patients with gastroenteropancreatic neuroendocrine tumors. J Nucl Med. 2018;59(3):452-458. doi:10.2967/jnumed.117.189712

88. Kong G, Callahan J, Hofman MS, et al. High clinical and morphologic response using ${ }^{90}$ Y-DOTA-octreotate sequenced with ${ }^{177}$ Lu-DOTA-octreotate induction peptide receptor chemoradionuclide therapy (PRCRT) for bulky neuroendocrine tumours. Eur J Nucl Med Mol Imaging. 2017;44(3):476-489. doi:10.1007/s00259-016-3527-x

89. van der Zwan WA, Brabander T, Kam BLR, et al. Salvage peptide receptor radionuclide therapy with [177 Lu-DOTA, Tyr 3]octreotate in patients with bronchial and gastroenteropancreatic neuroendocrine tumours. Eur J Nucl Med Mol Imaging. 2019;46 (3):704-717. doi:10.1007/s00259-018-4158-1

90. Goncalves I, Burbury K, Michael M, et al. Characteristics and outcomes of therapy-related myeloid neoplasms after Peptide Receptor Radionuclide Therapy (PRRT) for Metastatic Neuroendocrine Neoplasm (NEN): a single centre series. Neuroendocrinology. 2019;108:212.

91. Lin E, Chen T, Little A, et al. Safety and outcomes of (177) Lu-DOTATATE for neuroendocrine tumours: experience in New South Wales, Australia. Intern Med J. 2019;49(10):1268-1277. doi:10.1111/imj.14336

92. Gabriel M, Nilica B, Kaiser B, Virgolini IJ. Twelve-year follow-up after peptide receptor radionuclide therapy. $\mathrm{J} \mathrm{Nucl}$ Med. 2019;60(4):524-529. doi:10.2967/jnumed.118.215376

93. Vaughan E, Machta J, Walker M, et al. Retreatment with peptide receptor radionuclide therapy in patients with progressing neuroendocrine tumours: efficacy and prognostic factors for response. Br J Radiol. 2018;91(1091):20180041. doi:10.1259/ bjr.20180041

94. Thang SP, Lung MS, Kong G, et al. Peptide receptor radionuclide therapy (PRRT) in European Neuroendocrine Tumour Society (ENETS) grade 3 (G3) neuroendocrine neoplasia (NEN) - a single-institution retrospective analysis. Eur J Nucl Med $\mathrm{Mol}$ Imaging. 2018;45:262-277. 
95. Baum RP, Kulkarni HR, Singh A, et al. Results and adverse events of personalized peptide receptor radionuclide therapy with ${ }^{90}$ Yttrium and ${ }^{177}$ Lutetium in 1048 patients with neuroendocrine neoplasms. Oncotarget. 2018;9(24):16932-16950. doi:10.18632/oncotarget.24524

96. Yordanova A, Mayer K, Brossart P, et al. Safety of multiple repeated cycles of $177 \mathrm{Lu}$-octreotate in patients with recurrent neuroendocrine tumour. Eur J Nucl Med Mol Imaging. 2017;44 (7):1207-1214. doi:10.1007/s00259-017-3652-1

97. Katona BW, Roccaro GA, Soulen MC, et al. Efficacy of peptide receptor radionuclide therapy in a United States-based cohort of metastatic neuroendocrine tumor patients single-institution retrospective analysis. Pancreas. 2017;46(9):1121-1126. doi:10.1097/ mpa.0000000000000919

98. Mariniello A, Bodei L, Tinelli C, et al. Long-term results of PRRT in advanced bronchopulmonary carcinoid. Eur J Nucl Med Mol Imaging. 2016;43(3):441-452. doi:10.1007/s00259-015-3190-7

99. Brieau B, Hentic O, Lebtahi R, et al. High risk of myelodysplastic syndrome and acute myeloid leukemia after 177Lu-octreotate PRRT in NET patients heavily pretreated with alkylating chemotherapy. Endocr Relat Cancer. 2016;23(5):L17-L23. doi:10.1530/ERC-15-0543

100. Romer A, Seiler D, Marincek N, et al. Somatostatin-based radiopeptide therapy with [177Lu-DOTA]-TOC versus [90Y-DOTA] TOC in neuroendocrine tumours. Eur J Nucl Med Mol Imaging. 2014;41(2):214-222. doi:10.1007/s00259-013-2559-8

101. Sabet A, Ezziddin K, Pape U-F, et al. Long-term hematotoxicity after peptide receptor radionuclide therapy with $177 \mathrm{Lu}$-octreotate. $\mathrm{J} \mathrm{Nucl}$ Med. 2013;54(11):1857-1861. doi:10.2967/jnumed.112.119347

102. Kesavan M, Claringbold P, Turner JH. Hematological toxicity of combined lutetium-177 octreotate radiopeptide-chemotherapy $177 \mathrm{Lu}$-octreotate radiopeptide chemotherapy of gastroenteropancreatic neuroendocrine tumors followed long-term. Neuroendocrinology. 2014;99(2):283. doi:10.1159/000362558

103. Hentic O, Lebtahi R, Kwekkeboom DJ, et al. Delayed haematological toxicity in patients treated with 177 lu-octreotate peptide receptor radiotherapy (PRRT) for metastatic neuroendocrine tumors (NETs). Neuroendocrinology. 2014;99(3-4):282-283.

104. Richter S, Xenocostas A, Sanatani M et al. Evaluation of features and predictors for long term hematologic toxicity in neuroendocrine patients treated with peptide receptor radionuclide therapy. Neuroendocrinology. 2015;102(1-2):138.
105. Ginj M, Zhang H, Waser B, et al. Radiolabeled somatostatin receptor antagonists are preferable to agonists for in vivo peptide receptor targeting of tumors. Proc Natl Acad Sci U S A. 2006;103 (44):16436-16441. doi:10.1073/pnas.0607761103

106. Dalm SU, Nonnekens J, Doeswijk GN, et al. Comparison of the therapeutic response to treatment with a 177Lu-labeled somatostatin receptor agonist and antagonist in preclinical models. J Nucl Med. 2016;57(2):260-265. doi:10.2967/ jnumed.115.167007

107. Nicolas GP, Mansi R, McDougall L, et al. Biodistribution, pharmacokinetics, and dosimetry of 177 Lu-, 90 Y-, and 111 in-labeled somatostatin receptor antagonist OPS201 in comparison to the agonist $177 \mathrm{Lu}$-DOTATATE: the mass effect. $\mathrm{J} \mathrm{Nucl}$ Med. 2017;58(9):1435-1441. doi:10.2967/jnumed.117.191684

108. Claringbold PG, Turner JH. Pancreatic neuroendocrine tumor control: durable objective response to combination Lu-177-octreotatecapecitabine-temozolomide radiopeptide chemotherapy. Neuroendocrinology. 2016;103:432-439. doi:10.1159/000434723

109. Kong G, Thompson M, Collins M, et al. Assessment of predictors of response and long-term survival of patients with neuroendocrine tumour treated with peptide receptor chemoradionuclide therapy (PRCRT). Eur J Nucl Med Mol Imaging. 2014;41 (10):1831-1844. doi:10.1007/s00259-014-2788-5

110. Demaria S, Bhardwaj N, McBride WH, Formenti SC. Combining radiotherapy and immunotherapy: a revived partnership. Int J Radiat Oncol Biol Phys. 2005;63(3):655-666. doi:10.1016/ j.ijrobp.2005.06.032

111. Wu Y, Pfeifer A, Myschetzky R, et al. Induction of anti-tumor immune responses by peptide receptor radionuclide therapy with (177)Lu-DOTATATE in a murine model of a human neuroendocrine tumor. Diagnostics (Basel). 2013;3(4):344-355. doi:10.3390/diagnostics3040344

112. Morgenstern A, Apostolidis C, Kratochwil C, et al. An overview of targeted alpha therapy with 225 actinium and 213 bismuth. Curr Radiopharm. 2018;11(3):200-208. doi:10.2174/ 1874471011666180502104524

113. Navalkissoor S, Grossman A. Targeted alpha particle therapy for neuroendocrine tumours: the next generation of peptide receptor radionuclide therapy. Neuroendocrinology. 2019;108(3):256-264. doi: $10.1159 / 000494760$
OncoTargets and Therapy

\section{Publish your work in this journal}

OncoTargets and Therapy is an international, peer-reviewed, open access journal focusing on the pathological basis of all cancers, potential targets for therapy and treatment protocols employed to improve the management of cancer patients. The journal also focuses on the impact of management programs and new therapeutic

Submit your manuscript here: https://www.dovepress.com/oncotargets-and-therapy-journ agents and protocols on patient perspectives such as quality of life, adherence and satisfaction. The manuscript management system is completely online and includes a very quick and fair peer-review system, which is all easy to use. Visit http://www.dovepress.com/ testimonials.php to read real quotes from published authors. 